\title{
Comparison of computer-assisted navigated technology and conventional technology in unicompartmental knee arthroplasty: a meta-analysis
}

Keteng $\mathrm{Xu}^{1+}{ }^{+}$, Qun Chen ${ }^{2+}$, Qing Yan ${ }^{1 *}$, Qin Wang ${ }^{1 *}$ and Jun Sun ${ }^{1}$

\begin{abstract}
Background: Though unicompartmental knee arthroplasty (UKA) is a useful procedure to treat knee osteoarthritis, it remains a great controversial point as to if navigated systems are able to achieve better accuracy of limb alignment and greater clinic results. Current meta-analysis was conducted to explore if better clinical outcomes and radiographic outcomes could be acquired in the navigated system when compared with conventional procedures.

Methods: We identified studies in the online databases, including Medline, Embase, the Cochrane Library and Web of Science before May 2021. The PRISMA guidelines in this report were strictly followed. Our research was completed via Review Manager 5.4 software.

Results: Fourteen articles were included, involving 852 knees. The present meta-analysis displayed that the navigated system had remarkably improved outcomes in inliers of mechanical axis (MA) $(P<0.01)$, MA in the Kennedy's central zone (Zone $C)(P=0.04)$, inliers of the coronal femoral component $(P<0.01)$, inliers of the coronal tibial component $(P=0.005)$, inliers of the sagittal femoral component $(P=0.03)$, inliers of the sagittal tibial component $(P=0.002)$ and Range Of Motion (ROM) $(P=0.04)$. No significant differences were observed in Oxford Knee Score $(O K S)(P=0.15)$, American Knee Society Knee Score (KSS score) $(P=0.61)$ and postoperative complications $(P=0.73)$ between these 2 groups. Regarding operating time, the navigated group was 10.63 min longer in contrast to the traditional group.

Conclusion: Based on our research, the navigated system provided better radiographic outcomes and no significant difference in the risk of complications with longer surgical time than the conventional techniques. But no significant differences were found in functional outcomes. Because the included studies were small samples and short-term follow-up, high-quality RCTs with large patients and sufficient follow-up are required to identify the long-term effect of the navigated system.
\end{abstract}

Keywords: Navigation, Knee osteoarthritis, Unicompartmental knee arthroplasty, Meta-analysis

*Correspondence: hssrmyyyq@163.com; hssrmyywq@163.com ${ }^{\dagger}$ Keteng Xu and Chen Qun have contributed equally to this work 1 Department of Joint Surgery, Huangshan City People's Hospital, Huangshan, Anhui, China

Full list of author information is available at the end of the article

\section{Background}

Osteoarthritis (OA) is the main cause of disability in the elderly across the world, and it affects around $18 \%$ of females and $10 \%$ of males among people over 60 years old [1-3]. TKA and UKA are common surgical intervention for sufferers with end-stage knee OA [4-6]. Compared with TKA, UKA has less trauma, could keep 
the normal movement of knee joint, preserves cartilage and bone, has less postoperative complications, recovers quickly and is easier to repair when needed [7, 8]. But at the same time, the risk of malpositioning components is higher because of the limited visualization of anatomical landmarks. The failure of precise component positioning could result in rapid loosening of the component, promoted process of osteoarthritis to the opposite side of the knee and increased revision rate [9-13]. Nowadays, the computer navigated system has been frequently used in UKA procedures [14].

Computer navigated system began to emerge and used in TKA in the late 1990s. Some researches found computer navigated TKA could reduce outliers of alignment and provide good short-term outcomes [4]. Computer navigated system was introduced to UKA surgery by orthopedic surgeons in the early 2000s [14, 15]. This non-image-based system is designed to help orthopedists in improving the accuracy of bony resection and positioning component. By using the infrared camera and the dedicated software, orthopedists can calculate the rotation center of the hip, knee and ankle joints and determine the MA $[16,17]$. Previous meta-analyses summarized studies compared the two methods, and reported significantly better radiographic outcomes in patients undergone navigated UKA [18, 19]. But clinical outcomes were not reported. Besides, several new studies were published, including one RCT [7] and two studies [20,21] with average 9-year followup. Due to the need to discuss the clinical outcomes and the increase of high-level literature, it was the purpose of the present report to further assess the differences of radiological and clinical outcomes between the two methods.

\section{Methods}

The study protocol for the present report was accepted by the entire authors prior to the beginning of the literature search, and the protocol was published on the Internet at the PROSPERO (https://www.crd.york.ac.uk/prosp ero/) with the registration no. CRD42020172412.

\section{Inclusion and exclusion criteria}

The present report follows the inclusion standards below:

(1) Researches contrasted computer navigated UKA and traditional UKA. (2) Clinic or radiography results were not restricted for pooling. (3) Researches written in English were eligible. Researches were ruled out: (1) conferences, reviews, abstracts, case reports, sawbones or cadaveric knee researches. (2) Researches with insufficient data. (3) Redundant publication.

\section{Literature search}

Literature searches were finished to retrieve researches compared between the conventional group and navigational group in the UKA. The search terms were presented below: "navigat"," "Unicompartmental knee replacement," "unicompartmental knee arthroplasty," "unicondylar knee arthroplasty." Embase, Medline, Web of Science, Cochrane databases were retrieved to search relevant researches before May 2021.

\section{Data extraction}

The data below were separately abstracted from every selected researches by 2 researchers, including publication year, nation, the numbers of sufferers, age, gender, type of component, type of navigation system. The outcomes of our research include clinical outcomes and radiographic outcomes. Radiographic outcomes include inliers of MA (mechanical axis), MA in the Kennedy's zone $\mathrm{C}$, inliers of the coronal femur component, inliers of the sagittal femoral component, inliers of the coronal tibial component and inliers of the sagittal tibial component. The clinical outcomes include OKS scoring, KSS scoring, the WOMAC scoring, ROM, Pain scale (Visual Analogue Scale/Score, VAS), complications (infection, revision, deep venous thrombosis and so on), surgical time (minutes). Whenever there were disagreements, they would be solved via discussing with another researcher (XK).

\section{Quality evaluation}

The non-RCTs research quality was evaluated as per the Downs and Black [22] and the NOS [23] quality evaluation approach. An overall NOS scoring result was $9^{*}$, and when the NOS scoring result was $>6^{*}$, it was deemed as better quality. A greater scoring was deemed as higher quality. The 12-item scale was employed to evaluate the RCTs quality [24]. Every item was scored "Yes," "Unclear" or "No." When a trial with a scoring $>7$ "Yes" was deemed as high quality; $>4$ but $\leq 7$ was deemed as moderate quality, and $\leq 4$ was deemed as low quality. Any disagreement was solved by a third researcher.

\section{Statistical analysis}

Statistic data inhomogeneity was assessed via Cochran's $\mathrm{Q}$ statistics. If $\mathrm{Q}$ statistics $(P<0.10)$ was deemed as remarkable inhomogeneous among researches, a stochastic effect model was used; otherwise, a fix effect model was employed. If the parametric inhomogeneity was above $85 \%$, the gathering analysis would not be carried out. The outcomes of continuous data were applied to the average deviation with $95 \%$ CI. For dichotomous data, the OR was computed via the Mantel-Haenszel approach, average deviation and SMD were considered 
statistically significant at $P<0.05$. Data analyses were completed via Review Manager 5.4. Sensitivity analyses were finished to evaluate the outcomes via excluding eligible researches one by one.

\section{Results}

Study selection

By screening the titles and abstracts, 24 studies reaching the inclusion standards were reviewed for full-text screening. Posterior to the full-text eligibility evaluation, certain studies were excluded, as these researches focused on saw bones or cadaveric knees $[25,26]$ with insufficient data $[9,14,27-30]$, and partial data were used in other reports [15, 31]. Eventually, an overall 14 selected reports were described $[7,10,12,13,15,17,20,21,32-37]$. The features of the selected researches in the gathering analysis are presented in Table 1 . The navigational group involved 403 knees, and the traditional group involved 449 knees. The screening procedure is presented in Fig. 1.

\section{Quality of the included researches}

The present report comprises 12 non-RCT [10, 12, 13, 15, 17, 20, 21, 32-34, 36, 37], 2 RCTs [7, 35]. The level of evidence of all eligible studies was at least II level. From the perspective of Downs and Blacks scoring, the entire selected researches were above 15 . In NOS, all including non-RCTs had scored $\geq 6^{*}$. The non-RCTs report quality is shown in Table 2. The value of weighted kappa for the consensus on those researches between researchers was outstanding $(K=0.74)$. Two researches $[7,35]$ were high quality. The randomization approaches were clearly introduced in the two researches [7, 35]. Random distribution was sufficiently concealed in two researches [7, 35]. No RCTs offered the data of double blinding $[7,35]$. None displayed a binding of result evaluation $[7,35]$. The RCTs quality is presented in Table 3.

\section{Inliers of MA}

Eleven studies [7, 10, 13, 15, 20, 21, 32, 34-37] reported the rate of inliers of MA. There were 253 patients $(85.47 \%)$ in the navigated group and 217 sufferers $(69.33 \%)$ in the traditional group. No significant inhomogeneity was identified $\left(P=0.21 ; I^{2}=25 \%\right)$; hence, the fix effect model was employed. It displayed that the navigated group was remarkably more satisfactory than the conventional group in the rate of inliers of MA (OR 2.49, 95\% CI 1.66-3.71, $P<0.01$; Fig. 2).

\section{MA in the Kennedy's Zone C}

Five studies [20, 21, 32, 35, 37] reported MA in the Kennedy's Zone C. There were 83 sufferers $(60.14 \%)$ in the navigated group and 69 sufferers $(48.59 \%)$ in the traditional group. No significant inhomogeneity was identified
$\left(P=0.30 ; I^{2}=18 \%\right)$; thereby, the fix effect model was employed. It displayed that the navigated group had a remarkably more satisfactory result in the rate of MA in the Kennedy's Zone C (OR 1.65, 95\% CI 1.02-2.69, $P=0.04$; Fig. 3).

\section{Inliers of the coronal femoral component}

Six studies $[10,13,15,17,21,33]$ reported inliers of the coronal femoral component. There were 164 sufferers $(95.38 \%)$ in the navigated group and 181 sufferers $(83.03 \%)$ in the traditional group. There was no significant inhomogeneity among the researches $\left(P=0.95 ; I^{2}=0 \%\right)$, so the fix effect model was employed. It displayed that the navigated group was remarkably more satisfactory in the rate of inliers of the coronal femoral component than the conventional group (OR 4.80, 95\% CI 2.20 to 10.47, $P<0.01$; Fig. 4).

\section{Inliers of the sagittal femoral component}

Six studies $[10,13,15,17,21,33]$ reported inliers of the sagittal femoral component. There were 150 patients $(87.21 \%)$ in the navigated group and 150 sufferers (68.81\%) in the traditional group. Heterogeneity was remarkable in the studies $\left(P=0.04 .1 ; I^{2}=58 \%\right)$; thereby, the random effect model was employed. It displayed that the navigated group was remarkably more satisfactory in the rate of the sagittal femoral component than the traditional one (OR 3.03, 95\% CI 1.12 to 8.25, $P=0.03$; Fig. 5).

\section{Inliers of the coronal tibial component}

Six studies [10,13, 15, 17, 21, 33] reported inliers of the coronal tibial component. There were 158 patients (91.86\%) in the navigated group and 185 sufferers $(84.86 \%)$ in the traditional group. No significant inhomogeneity was identified $\left(P=0.76 ; I^{2}=0 \%\right)$; thereby, the fix effect model was employed. It displayed that the navigated group was remarkably more satisfactory in the rate of inliers of the coronal tibial component than the traditional one (OR 2.76, 95\% CI 1.37 to $5.58, P=0.005$; Fig. 6).

\section{Inliers of the sagittal tibial component}

Six studies [10,13,15, 17, 21, 33] reported inliers of the sagittal tibial component. There were 147 patients $(85.47 \%)$ in the navigated group and 154 sufferers (70.64\%) in the traditional group. No remarkable inhomogeneity was identified $\left(P=0.31 ; I^{2}=15 \%\right)$; thereby, the fix effect model was employed. It displayed that the navigated group was remarkably more satisfactory in the rate of inliers of the sagittal tibial component than the traditional one (OR 2.30, 95\% CI 1.37 to $3.86, P=0.002$; Fig. 7). 
Table 1 Summary of included studies (a)

\begin{tabular}{|c|c|c|c|c|c|c|c|}
\hline References & Country & Type & $\begin{array}{l}\text { Age, Mean (SD or } \\
\text { range) }\end{array}$ & Gender (F/M) & Keen (n) & $\begin{array}{l}\text { Navigated } \\
\text { system }\end{array}$ & Type of prosthesis \\
\hline Song et al. [21] & Korea & Cohort study & $\begin{array}{l}\text { Navigated group: } \\
63.6 \text { (50-79) } \\
\text { Conventional } \\
\text { group: } 64.3 \text { (52-81) }\end{array}$ & $\begin{array}{l}\text { Navigated group: } \\
33 / 1 \\
\text { Conventional } \\
\text { group: } 32 / 2\end{array}$ & $\begin{array}{l}\text { Navigated group: } \\
34 \\
\text { Conventional } \\
\text { group: } 34\end{array}$ & OrthoPilot & Zimmer \\
\hline Zhang et al. [7] & China & $\begin{array}{l}\text { Randomized con- } \\
\text { trolled trial }\end{array}$ & $\begin{array}{l}\text { Navigated group: } \\
62.4(5.62) \\
\text { Conventional } \\
\text { group: } 61.9(6.11)\end{array}$ & $\begin{array}{l}\text { Navigated group: } \\
22 / 18 \\
\text { Conventional } \\
\text { group: } 22 / 19\end{array}$ & $\begin{array}{l}\text { Navigated group: } \\
40 \\
\text { Conventional } \\
\text { group: } 41\end{array}$ & BrainLAB & Zimmer \\
\hline Manzotti et al. [12] & Italy & Case-control study & $\begin{array}{l}\text { Navigated group: } \\
70.9(7.8) \\
\text { Conventional } \\
\text { group: } 71.3 \text { (6.8) }\end{array}$ & $\begin{array}{l}\text { Navigated group: } \\
18 / 13 \\
\text { Conventional } \\
\text { group: } 18 / 13\end{array}$ & $\begin{array}{l}\text { Navigated group: } \\
31 \\
\text { Conventional } \\
\text { group: } 31\end{array}$ & OrthoPilot & DePuy \\
\hline $\begin{array}{l}\text { Valenzuela et al. } \\
\text { [37] }\end{array}$ & USA & Case-control study & $\begin{array}{l}\text { Navigated Mean: } \\
65 \text { (48-81) } \\
\text { Conventional } \\
\text { Mean: } 67.3 \text { (49-81) }\end{array}$ & $\begin{array}{l}\text { Navigated group: } \\
28 / 28 \\
\text { Conventional } \\
\text { group: } 36 / 30\end{array}$ & $\begin{array}{l}\text { Navigated group: } \\
56 \\
\text { Conventional } \\
\text { group: } 66\end{array}$ & Praxim & Zimmer \\
\hline Weber et al. [17] & Austria & Case-control study & $\begin{array}{l}\text { Navigated Mean: } \\
67.9(44-81.4) \\
\text { Conventional } \\
\text { Mean: } 69.1 \\
(53.1-79.5)\end{array}$ & $\begin{array}{l}\text { Navigated group: } \\
11 / 9 \\
\text { Conventional } \\
\text { group: } 10 / 10\end{array}$ & $\begin{array}{l}\text { Navigated group: } \\
20 \\
\text { Conventional } \\
\text { group: } 20\end{array}$ & OrthoPilot & Univation \\
\hline Konyves et al. [20] & Australia & Case-control study & $\begin{array}{l}\text { Navigated group: } \\
59(41-78) \\
\text { Conventional } \\
\text { group: } 61 \text { (44-71) }\end{array}$ & $N R$ & $\begin{array}{l}\text { Navigated group: } \\
15 \\
\text { Conventional } \\
\text { group: } 15\end{array}$ & Stryker & Sulzer \\
\hline Jung et al. [13] & Korea & Cohort study & $\begin{array}{l}\text { Navigated group: } \\
59(51-76) \\
\text { Conventional } \\
\text { group: } 56 \text { (46-68) }\end{array}$ & $\begin{array}{l}\text { Navigated group: } \\
13 / 4 \\
\text { Conventional } \\
\text { group: } 15 / 10\end{array}$ & $\begin{array}{l}\text { Navigated group: } \\
23 \\
\text { Conventional } \\
\text { group: } 29\end{array}$ & Stryker & Oxford \\
\hline Lim et al. [35] & Australia & $\begin{array}{l}\text { Randomized con- } \\
\text { trolled trial }\end{array}$ & $\begin{array}{l}\text { Navigated group: } \\
60 \text { (50-78) } \\
\text { Conventional } \\
\text { group: } 72 \text { (46-85) }\end{array}$ & $\begin{array}{l}\text { Navigated group: } \\
15 / 15 \\
\text { Conventional } \\
\text { group: } 11 / 10\end{array}$ & $\begin{array}{l}\text { Navigated group: } \\
30 \\
\text { Conventional } \\
\text { group: } 21\end{array}$ & OrthoPilot & FREEDOM \\
\hline $\begin{array}{l}\text { Rosenberger et al. } \\
{[10]}\end{array}$ & Austria & Case-control study & $\begin{array}{l}\text { Navigated group: } \\
62.9(6.48) \\
\text { Conventional } \\
\text { group: } 66.65 \text { (6.48) }\end{array}$ & $\begin{array}{l}\text { Navigated group: } \\
13 / 7 \\
\text { Conventional } \\
\text { group: } 9 / 11\end{array}$ & $\begin{array}{l}\text { Navigated group: } \\
20 \\
\text { Conventional } \\
\text { group: } 20\end{array}$ & TREON plus & Oxford \\
\hline Jenny et al. [33] & France & Case-control study & $\begin{array}{l}\text { Navigated Mean: } \\
65.6(48-84) \\
\text { Conventional } \\
\text { Mean: } 64.5 \text { (44-88) }\end{array}$ & $\begin{array}{l}\text { Navigated group: } \\
34 / 15 \\
\text { Conventional } \\
\text { group: 54/33 }\end{array}$ & $\begin{array}{l}\text { Navigated group: } \\
49 \\
\text { Conventional } \\
\text { group: } 87\end{array}$ & OrthoPilot & Aesculap \\
\hline Keene et al. [34] & Australia & Cohort study & NR & NR & $\begin{array}{l}\text { Navigated Mean: } \\
20 \\
\text { Conventional } \\
\text { Mean: } 20\end{array}$ & $\begin{array}{l}\text { Ci(DePuy/Brain- } \\
L A B)\end{array}$ & DePuy \\
\hline Perlick et al. [36] & Denmark & Cohort study & $\begin{array}{l}\text { Navigated Mean: } \\
65 \text { (49-73) } \\
\text { Conventional } \\
\text { Mean: } 67 \text { (45-74) }\end{array}$ & $\begin{array}{l}\text { Navigated group: } \\
14 / 6 \\
\text { Conventional } \\
\text { group: } 12 / 8\end{array}$ & $\begin{array}{l}\text { Navigated group: } \\
20 \\
\text { Conventional } \\
\text { group: } 20\end{array}$ & $\begin{array}{l}\text { Ci(DePuy/Brain- } \\
L A B)\end{array}$ & DePuy \\
\hline $\begin{array}{l}\text { Cossey and Sprig- } \\
\text { gins [32] }\end{array}$ & UK & Cohort study & $\begin{array}{l}\text { Navigated group: } \\
55(41-78) \\
\text { Conventional } \\
\text { group: } 57 \text { (42-74) }\end{array}$ & $\begin{array}{l}\text { Navigated group: } \\
8 / 6 \\
\text { Conventional } \\
\text { group: } 10 / 4\end{array}$ & $\begin{array}{l}\text { Navigated group: } \\
15 \\
\text { Conventional } \\
\text { group: } 15\end{array}$ & Stryker & $\begin{array}{l}\text { Stryker-Howmedica, } \\
\text { Sulzer }\end{array}$ \\
\hline $\begin{array}{l}\text { Jenny and Boeri } \\
\text { [15] }\end{array}$ & France & Case-control study & NR & NR & $\begin{array}{l}\text { Navigated Mean: } \\
30 \\
\text { Conventional } \\
\text { Mean: } 30\end{array}$ & OrthoPilot & Aesculap \\
\hline
\end{tabular}

NR, no report 


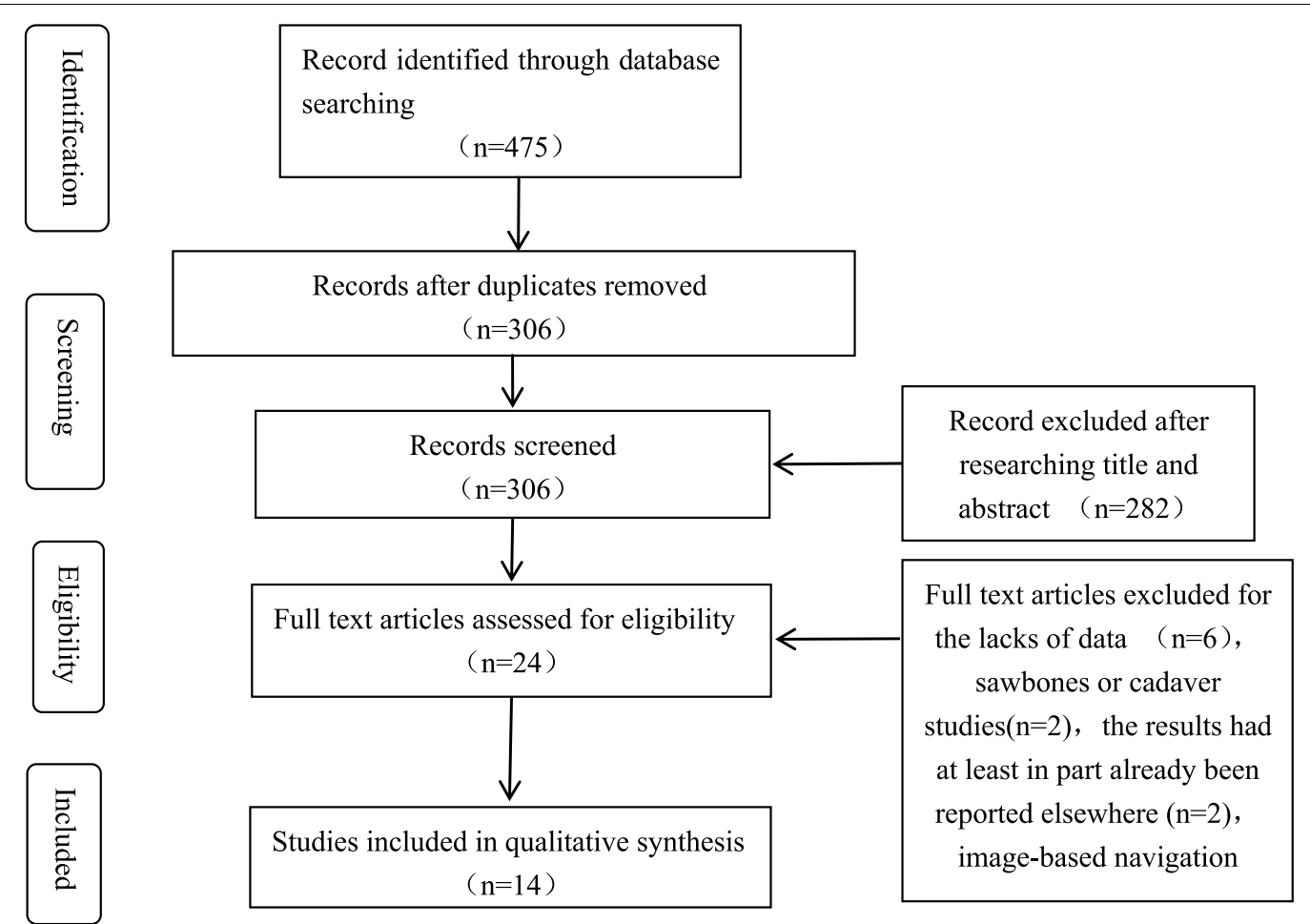

Fig. 1 Flowchart of the study selection procedure

\section{OKS score}

Two studies [20,32] contrasted the OKS score. These data were gathered for analyses. No remarkable inhomogeneity existed among the researches $\left(P=0.40 ; I^{2}=0\right)$; hence, the fix effect model was employed. It displayed no remarkable diversity in the OKS score between these 2 groups $(\mathrm{MD}=-1.17,95 \% \mathrm{CI}-2.74$ to $0.40, P=0.15$; Fig. 8).

\section{KSS score}

Three studies [7, 17, 35] compared the KSS score. These data were gathered for analyses. No remarkable inhomogeneity existed among the researches $\left(P=0.83 ; I^{2}=0\right)$; hence, the fix effect model was employed. There was not remarkable diversity in the KSS score between these 2 groups $(\mathrm{MD}=-0.02,95 \% \mathrm{CI}-1.18$ to $1.15, P=0.61$; Fig. 9).

\section{WOMAC score}

In the articles we included two reported results of the WOMAC score [12, 21]. The inhomogeneity was above $85 \%\left(I^{2}=95 \%\right)$; for that reason, the overall effects of this parameter were not implemented (Table 4).

\section{ROM}

Two studies compared [7, 21] the ROM. Thereby, our team accepted them for the gathering analysis. No significant inhomogeneity was identified between the researches $\left(P=0.96 ; I^{2}=0 \%\right)$; hence, the fix effect model was employed. The gathered outcomes displayed that the navigational group exhibited a remarkable increase in $\operatorname{ROM}(M D=2.44,95 \%$ CI 0.38 to $4.51, P=0.04$; Fig. 10$)$.

\section{VAS}

Two researches $[7,12]$ involved the VAS. The inhomogeneity was above $85 \%\left(I^{2}=87 \%\right)$; for that reason, the overall effects of this parameter were not implemented.

\section{Complications}

Ten studies [7, 12, 13, 15, 17, 21, 32, 34-36] displayed complicating diseases. The navigated group had 8 sufferers $(2.76 \%)$, and the traditional group had 7 sufferers $(2.42 \%)$. No significant inhomogeneity was identified $\left(P=0.91 ; I^{2}=0 \%\right)$; hence, the fix effect model was employed. It displayed no significant diversity in the rate of complications between these 2 groups (OR 1.20, 95\% CI 0.41 to $3.52, P=0.73$; Fig. 11 ). 
Table 2 Summary of included studies (b)

\begin{tabular}{|c|c|c|c|c|c|c|}
\hline References & Follow-up & $\begin{array}{l}\text { Functional } \\
\text { evaluation }\end{array}$ & $\begin{array}{l}\text { Mechanical } \\
\text { axis }\end{array}$ & $\begin{array}{l}\text { Positioning of } \\
\text { the prosthesis }\end{array}$ & $\begin{array}{l}\text { Complications } \\
\text { (Navigated group } \\
\text { vs. conventional } \\
\text { group) }\end{array}$ & $\begin{array}{l}\text { Operating time (SD) } \\
\text { [Min] }\end{array}$ \\
\hline Song et al. [21] & 9 years & HSS, WOMAC & Yes & Yes & $\begin{array}{l}1 \text { revision/2 knees } \\
\text { were revised }\end{array}$ & $N R$ \\
\hline Zhang et al. [7] & 2 years & KSS, KSFS & Yes & Yes & $\begin{array}{l}\text { No case of complica- } \\
\text { tions }\end{array}$ & $\begin{array}{l}\text { Navigated group: } \\
59.4 \pm 6.1 \\
\text { Conventional group: } \\
62.1 \pm 5.5\end{array}$ \\
\hline Manzotti et al. [12] & 6 months & WOMAC, IKS & No & Yes & $\begin{array}{l}\text { No case of complica- } \\
\text { tions }\end{array}$ & $\begin{array}{l}\text { Navigated group: } \\
47.4 \pm 6.1 \\
\text { Conventional group: } \\
35.4 \pm 4.4\end{array}$ \\
\hline Valenzuela et al. [37] & NR & NR & Yes & Yes & NR & $N R$ \\
\hline Weber et al. [17] & 18 months & KSS & $N R$ & $N R$ & $\begin{array}{l}2 \text { patients were } \\
\text { revised to a TKA } 1 \\
\text { patient was revised } \\
\text { to a TKA }\end{array}$ & $\begin{array}{l}\text { Navigated group: } \\
126.2 \pm 21.25 \\
\text { Conventional group: } \\
109.4 \pm 18.25\end{array}$ \\
\hline Konyves et al. [20] & $\begin{array}{l}\text { Navigated group: } \\
6.9 \text { years Conven- } \\
\text { tional group: } 8.9 \text { years }\end{array}$ & OKS & No & No & NR & $N R$ \\
\hline Jung et al. [13] & NR & NR & No & Yes & $\begin{array}{l}\text { One case of tibial side } \\
\text { pin site infection/one } \\
\text { case of intraoperative } \\
\text { fracture, one case of } \\
\text { infection }\end{array}$ & $\begin{array}{l}\text { Navigated group: } 98 \\
\text { Conventional group: } 82\end{array}$ \\
\hline Lim et al. [35] & 1 year & KSS & No & NR & $\begin{array}{l}\text { No case of complica- } \\
\text { tions }\end{array}$ & NR \\
\hline $\begin{array}{l}\text { Rosenberger et al. } \\
\text { [10] }\end{array}$ & NR & NR & Yes & NR & $\begin{array}{l}\text { No case of complica- } \\
\text { tions }\end{array}$ & $\begin{array}{l}\text { Navigated group: } \\
81.8 \pm 11.08 \\
\text { Conventional group: } \\
70.85 \pm 14.86\end{array}$ \\
\hline Jenny et al. [33] & NR & NR & NR & Yes & $\begin{array}{l}\text { No case of complica- } \\
\text { tions }\end{array}$ & NR \\
\hline Keene et al. [34] & $N R$ & NR & NR & NR & $\begin{array}{l}\text { No case of complica- } \\
\text { tions }\end{array}$ & $\begin{array}{l}\text { Navigated group: } 70 \\
\text { Conventional group: } 53\end{array}$ \\
\hline Perlick et al. [36] & NR & NR & Yes & NR & $\begin{array}{l}\text { No case of complica- } \\
\text { tions }\end{array}$ & $\begin{array}{l}\text { Navigated group: } \\
77 \pm 14 \\
\text { Conventional group: } \\
58 \pm 11\end{array}$ \\
\hline $\begin{array}{l}\text { Cossey and Spriggins } \\
{[32]}\end{array}$ & 17 months & OKS & Yes & Yes & $\begin{array}{l}2 \text { deep venous } \\
\text { thrombosis, } 1 \text { wound } \\
\text { infection/1 deep } \\
\text { venous thrombosis } \\
\text { and } 1 \text { superficial } \\
\text { wound infection }\end{array}$ & $\begin{array}{l}\text { Navigated group: } 81 \\
\text { Conventional group: } 58\end{array}$ \\
\hline Jenny and Boeri [15] & 3 months & NR & Yes & Yes & $N R$ & $\begin{array}{l}\text { Navigated group: } 86 \\
\text { Conventional group: } 67\end{array}$ \\
\hline
\end{tabular}

KSS, Knee Society Score; WOMAC, Western Ontario and McMaster Osteoarthritis Index; IKS, International Knee Society; OKS, Oxford Knee Score

Yes, Better positioning of the prosthesis/mechanical axis through navigation; No, no difference; NR, no report

\section{Surgical time (minutes)}

Five articles $[7,10,12,17,36]$ reported the outcomes of surgical time. The navigated group was 10.63 min longer in contrast to the traditional one. The inhomogeneity was above $85 \%\left(I^{2}=95 \%\right)$; for that reason, the overall effects of this parameter were not implemented.

\section{Sensitivity analyses}

One research was individual removed every time to identify the influence on the gathered MD or OR. The outcomes displayed that no research was able to remarkably influence the gathered MD or OR in this gathering analysis. 
Table 3 Description of the quality of included non-RCTs studies

\begin{tabular}{|c|c|c|c|c|c|c|c|c|c|}
\hline \multirow[t]{2}{*}{ References } & \multirow[t]{2}{*}{ Country } & \multirow[t]{2}{*}{ Type } & \multirow{2}{*}{$\begin{array}{l}\text { Level of } \\
\text { evidence }\end{array}$} & \multirow{2}{*}{$\begin{array}{l}\text { Study quality } \\
\text { Downs and Black } \\
\text { Score }\end{array}$} & \multicolumn{5}{|l|}{ NOS Scale } \\
\hline & & & & & Selection & Comparability & Expose & Outcome & Total score \\
\hline Song et al. [21] & Korea & Cohort study & III & 16 & $* * *$ & $* *$ & - & $* *$ & $* * * * * * *$ \\
\hline Manzotti et al. [12] & Italy & $\begin{array}{l}\text { Case-control } \\
\text { study }\end{array}$ & $\|$ & 21 & $* * *$ & $* *$ & $* *$ & - & $* * * * * * *$ \\
\hline $\begin{array}{l}\text { Valenzuela et al. } \\
\text { [37] }\end{array}$ & USA & $\begin{array}{l}\text { Case-control } \\
\text { study }\end{array}$ & $\|$ & 17 & $* * *$ & $* *$ & * & - & $* * * * * *$ \\
\hline Weber et al. [17] & Austria & $\begin{array}{l}\text { Case-control } \\
\text { study }\end{array}$ & $\|$ & 23 & $* *$ & $*$ & $* *$ & - & $* * * * * *$ \\
\hline Konyves et al. [20] & Australia & $\begin{array}{l}\text { Case-control } \\
\text { study }\end{array}$ & $\|$ & 19 & $* * *$ & $* *$ & $* *$ & - & $* * * * * * *$ \\
\hline Jung et al. [13] & Korea & Cohort study & III & 20 & $* * *$ & $* *$ & - & $* *$ & $* * * * * * *$ \\
\hline $\begin{array}{l}\text { Rosenberger et al. } \\
\text { [10] }\end{array}$ & Austria & $\begin{array}{l}\text { Case-control } \\
\text { study }\end{array}$ & $\|$ & 16 & $* * *$ & $* *$ & $* *$ & - & $* * * * * * *$ \\
\hline Jenny et al. [33] & South-Korea & $\begin{array}{l}\text { Case-control } \\
\text { study }\end{array}$ & $\|$ & 16 & $* *$ & $* *$ & $* *$ & - & $* * * * * *$ \\
\hline Keene et al. [34] & France & Cohort study & III & 17 & $* * *$ & * & - & $* *$ & $* * * * * *$ \\
\hline Perlick et al. [36] & Australia & Cohort study & $\|$ & 18 & $* * *$ & $* *$ & - & $*$ & $* * * * * *$ \\
\hline $\begin{array}{l}\text { Cossey and Sprig- } \\
\text { gins [32] }\end{array}$ & Denmark & Cohort study & $\|$ & 19 & $* * *$ & $*$ & - & $* *$ & $* * * * * *$ \\
\hline $\begin{array}{l}\text { Jenny and Boeri } \\
\text { [15] }\end{array}$ & UK & $\begin{array}{l}\text { Case-control } \\
\text { study }\end{array}$ & III & 18 & $* * *$ & $* *$ & $* *$ & - & $* * * * * * *$ \\
\hline
\end{tabular}

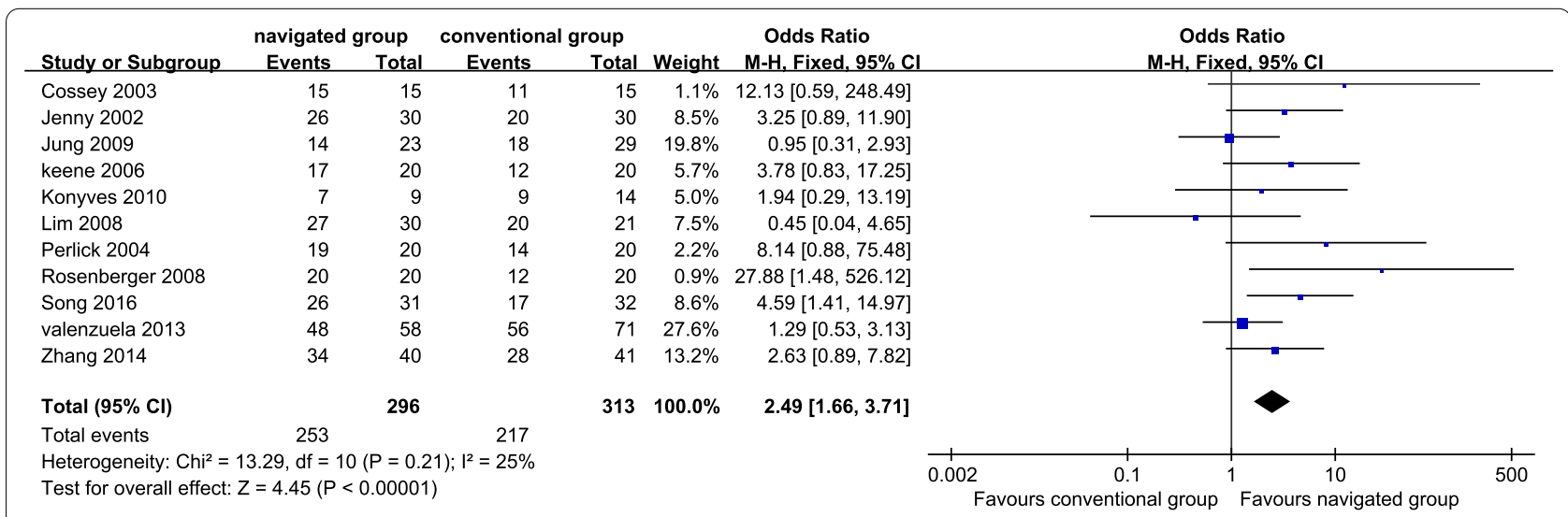

Fig. 2 Forest plot diagram showed the mean difference in Oxford Knee Score (OKS score) between navigated group and conventional group

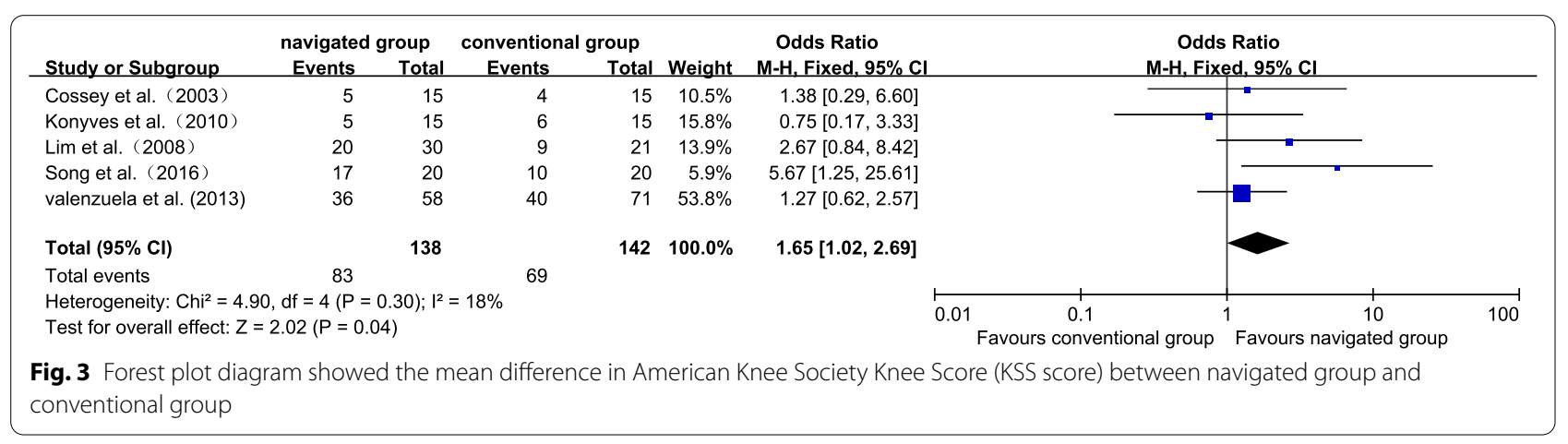




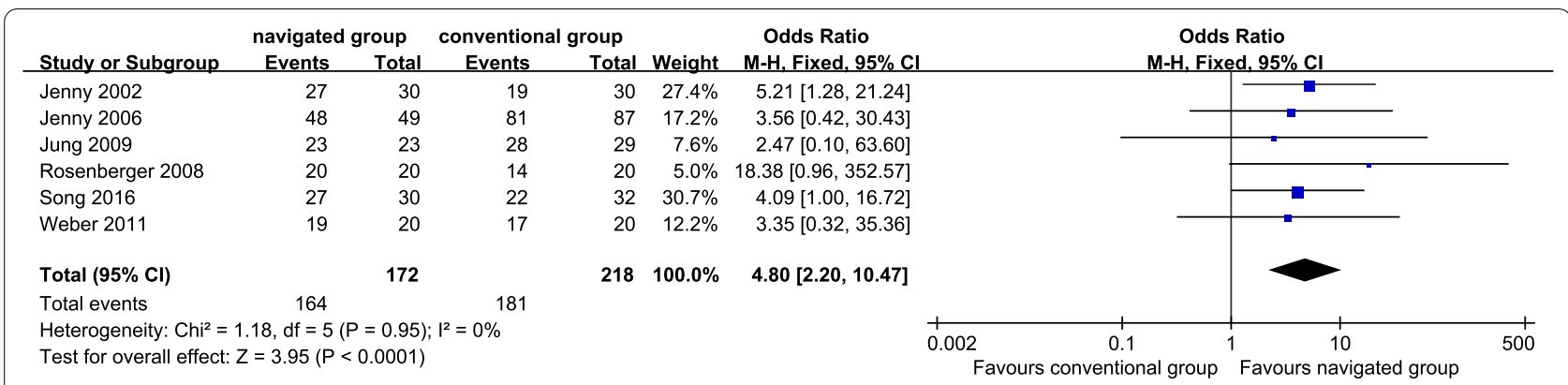

Fig. 4 Forest plot diagram showed the mean difference in Range Of Motion (ROM) between navigated group and conventional group

\begin{tabular}{|c|c|c|c|c|c|c|c|c|c|c|c|}
\hline \multirow[b]{2}{*}{ Study or Subgroup } & \multicolumn{2}{|c|}{ navigated group } & \multicolumn{2}{|c|}{ conventional group } & \multirow{2}{*}{ Weight } & \multirow{2}{*}{$\begin{array}{l}\text { Odds Ratio } \\
\text { M-H, Random, } 95 \% \mathrm{Cl}\end{array}$} & \multirow{2}{*}{\multicolumn{5}{|c|}{$\begin{array}{c}\text { Odds Ratio } \\
\text { M-H, Random, } 95 \% \mathrm{Cl}\end{array}$}} \\
\hline & Events & Total & Events & Total & & & & & & & \\
\hline Jenny 2002 & 27 & 30 & 19 & 30 & $19.5 \%$ & $5.21[1.28,21.24]$ & & & & & \\
\hline Jenny 2006 & 40 & 49 & 57 & 87 & $25.8 \%$ & $2.34[1.00,5.46]$ & & & & & \\
\hline Jung 2009 & 23 & 23 & 21 & 29 & $8.6 \%$ & $18.58[1.01,341.62]$ & & & & & \\
\hline Rosenberger 2008 & 20 & 20 & 14 & 20 & $8.4 \%$ & $18.38[0.96,352.57]$ & & & & & \\
\hline Song 2016 & 27 & 30 & 22 & 32 & $19.5 \%$ & $4.09[1.00,16.72]$ & & & & & \\
\hline Weber 2011 & 13 & 20 & 17 & 20 & $18.2 \%$ & $0.33[0.07,1.52]$ & & & - & & \\
\hline Total $(95 \% \mathrm{Cl})$ & & 172 & & 218 & $100.0 \%$ & $3.03[1.12,8.25]$ & & & & & \\
\hline Total events & 150 & & 150 & & & & & & & & \\
\hline $\begin{array}{l}\text { Heterogeneity: } \mathrm{Tau}^{2}= \\
\text { Test for overall effect: }\end{array}$ & $\begin{array}{l}.82 ; \mathrm{Chi}^{2}= \\
=2.17(\mathrm{P}\end{array}$ & $\begin{array}{l}\text { 1.93, df } \\
0.03)\end{array}$ & $=5(P=0.04$ & $=58 \%$ & & & $\begin{array}{r}0.005 \\
\mathrm{Fa}\end{array}$ & ${ }_{\text {ventional group }}^{1}$ & 1 Favours na & $\begin{array}{l}10 \\
\text { vigated group }\end{array}$ & 200 \\
\hline
\end{tabular}

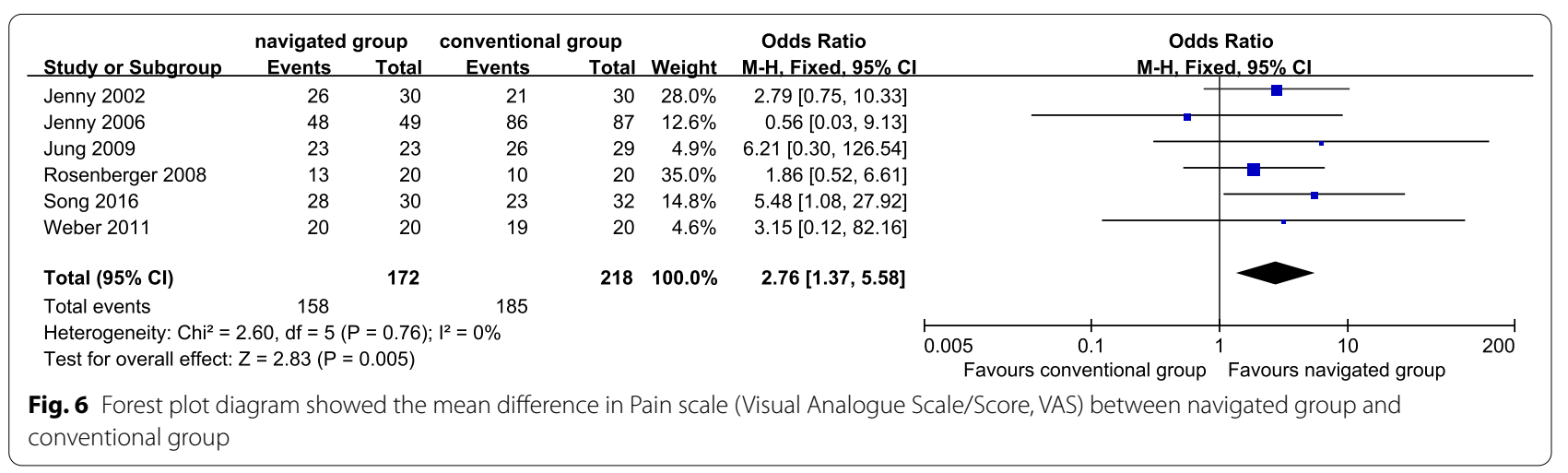

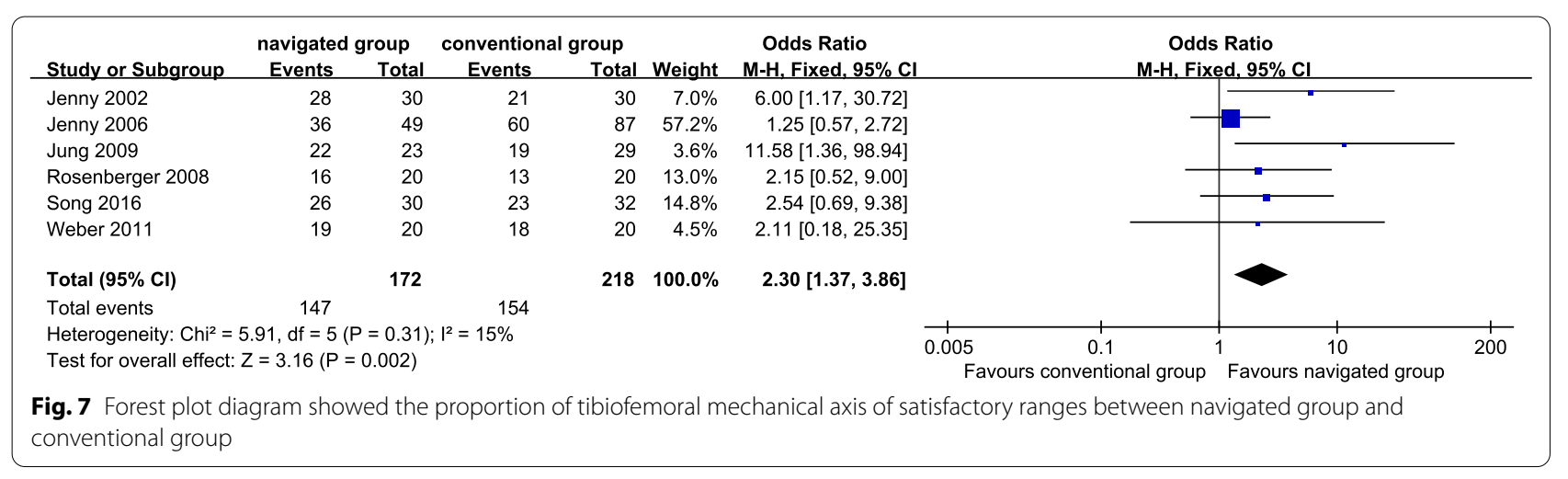




\begin{tabular}{|c|c|c|c|c|c|c|c|c|c|}
\hline \multirow[b]{2}{*}{ Study or Subgroup } & \multicolumn{3}{|c|}{ navigated group } & \multicolumn{3}{|c|}{ conventional group } & & \multirow{2}{*}{$\begin{array}{l}\text { Mean Difference } \\
\text { IV, Fixed, } 95 \% \mathrm{Cl}\end{array}$} & \multirow{2}{*}{$\begin{array}{l}\text { Mean Difference } \\
\text { IV.Fixed. } 95 \% \mathrm{CI}\end{array}$} \\
\hline & Mean & SD & Total & Mean & SD & Total & Weight & & \\
\hline Cossey et al. (2003) & 17 & 2.5 & 15 & 18 & 2 & 15 & $94.2 \%$ & $-1.00[-2.62,0.62]$ & $-F$ \\
\hline Konyves et al. (2010) & 35.5 & 9.2368 & 9 & 39.4 & 6.1484 & 23 & $5.8 \%$ & $-3.90[-10.44,2.64]$ & \\
\hline Total $(95 \% \mathrm{Cl})$ & & & 24 & & & 38 & $100.0 \%$ & $-1.17[-2.74,0.40]$ & \\
\hline \multicolumn{8}{|c|}{$\begin{array}{l}\text { Heterogeneity: Chi }{ }^{2}=0.71, d f=1(P=0.40) ; I^{2}=0 \% \\
\text { Test for overall effect: } Z=1.46(P=0.15)\end{array}$} & & $\begin{array}{ccccc}-10 & -5 & 0 & 5 & 10 \\
\text { Favours conventional group } & \text { Favours navigated group }\end{array}$ \\
\hline
\end{tabular}

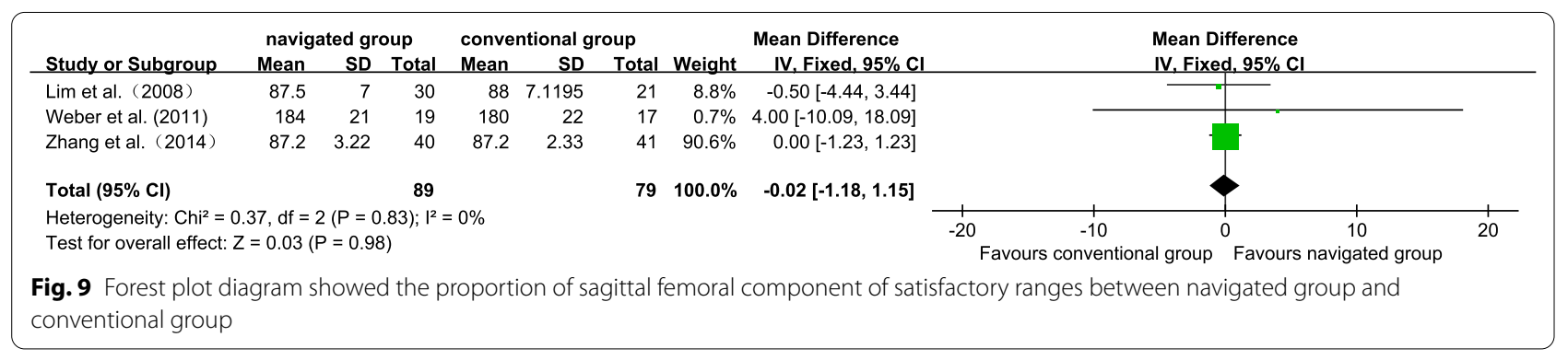

\section{Discussion}

The discovery of our gathering analysis was that using the navigation system significantly improved the precision of rebuilding the MA and placing components in UKA. Regarding clinical outcomes, ROM of navigation group was significantly better than that of conventional group. Besides, the operative duration of the navigated group was 10.63 min longer in contrast to the traditional one. But no remarkable diversity was identified in the OKS scoring, the KSS scoring and complications.

It is generally accepted that the accuracy of radiographic outcomes is crucial for knee pain relief, implant survival and long-term functional outcomes [11, 18, 29, $38,39]$. Our meta-analysis evaluated radiographic outcomes by the inliers of MA, the position of femoral components, the position of tibial components and the rate of MA in the Zone C. Unlike previous studies [18, 19], our study added the rate of MA in the Zone $C$, which is recognized as an acceptable range for optimizing longterm results, to further evaluate the accuracy of radiographic outcomes between the two methods [40]. The present gathering analysis discovered that the navigated system remarkably reduced the risk of MA outliers and components, improved the rate of MA falling in Zone C. Song et al. also found the use of the navigated system contributed to significantly improved rate of desired MA and components placement over an average 9-year follow-up [21]. Although some researchers have found no significant difference in the radiographic outcomes, they have identified possible reasons for the conflicting outcomes, such as small samples, short follow-up, inaccurate navigated system and software incompatibility [12, 35]. However, these studies also favored using the navigated system when inexperienced orthopedists performed UKA.

There is much debate about the ideal position of the tibial prosthesis. Some orthopedists are happy to maintain neutral correction of tibial axis, some insist on slight under correction, while certain orthopedists are favor to reconstruct the initial anatomy structure without worrying about the tibial axis [11, 14]. In the included studies, the definition of the ideal tibial slope is also different. It was 3-degree in Song's study [21], 5-degree in Rosenberger's study [10] and 7-degree posterior to the 2-degree anterior in Weber's study [17], whereas Perlick et al. favored tibial slope adapted according to individual patient's situation [36]. Researches are required to discuss the ideal position of the tibial prosthesis. Determining the ideal position of the tibial prosthesis can more accurately compare the advantages and disadvantages of the two groups.

Functional outcomes are important indicators to evaluate the effect of UKA. Our studies evaluated functional outcomes based on the OKS score, KSS score and ROM. Although the navigated group had significantly better outcome in ROM, no remarkable diversity existed in OKS score and KSS score between the two groups. The introduction of the navigated system bases on the assumption that the optimal MA and components location are closely associated with satisfying functional outcomes. However, our results contradicted the assumption. The small sample of some included studies may contribute to it. Only 


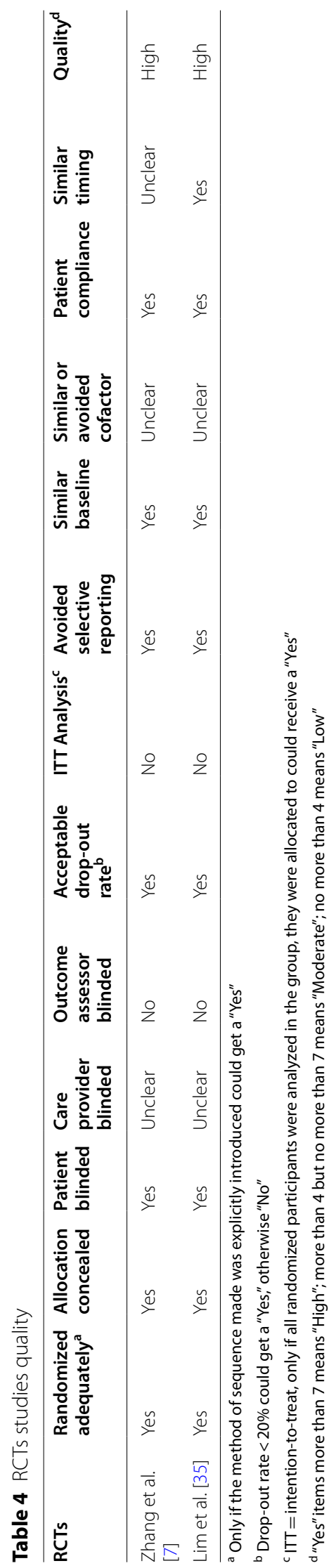




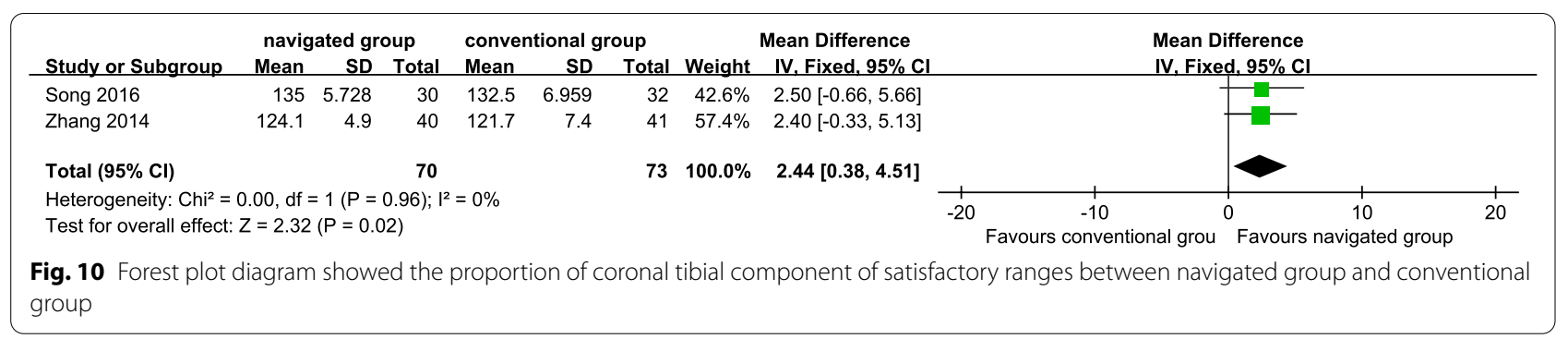

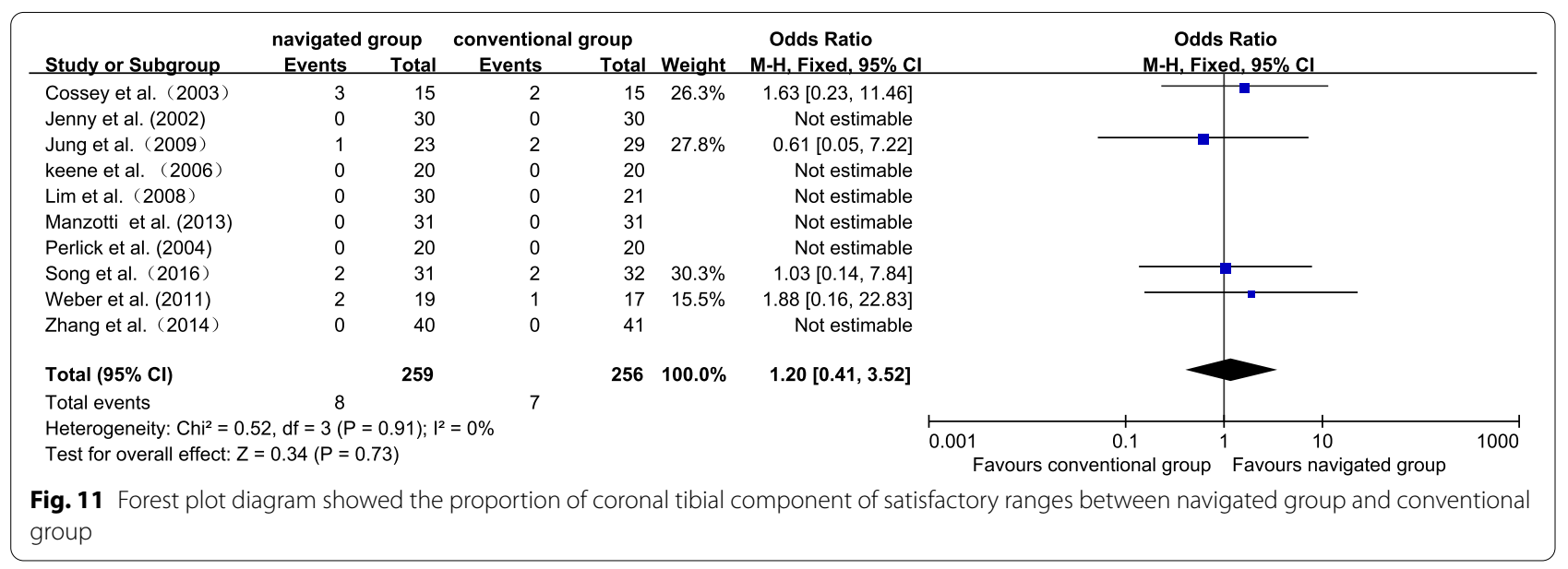

two studies [20,32] reported the OKS and three studies $[7,17,35]$ reported the KSS. Besides, the effect of fine MA on the postoperative function needs a long period to be obvious [7, 17, 20,32,35]. Song et al. had reported the navigated group had more accuracy radiographic outcomes, significantly better results in functional scaleHospital for Special Surgery (HSS) and pain scale scores were found over an average 9-year follow-up. Saragaglia et al. also supported the navigated system had better outcomes in a long-term follow-up [14].

In our meta-analysis, no remarkable diversity existed in the rate of postoperative complicating diseases (infection, revision, deep venous thrombosis and so on) between these 2 groups. Chona et al. contrasted the ratio of complications between the two methods using the Medicare database (included 9,228 UKAs) [16], and they also found no significant difference at 30 days, 90 days and 2 years. Therefore, we speculated there may be no remarkable diversity between these 2 methods in the prevalence of complications during short-term follow-up. But it is hard to tell the difference between long-term follow-up, because most of the studies included were short-term follow-up. A mean follow-up time of 10 years may be sufficient to determine whether there is a diversity in the incidence of complicating diseases between these 2 groups [41].
Some studies have reported on the disadvantages of the navigation system, including the longer operating time $[7,10,12,17,36]$. Certain extra steps to fasten the tracking device to the limb and register anatomical signs may be the cause of longer surgical time $[12,21]$. But the navigated system has the potential advantage of improving the precision of implant positioning. Thus, the additional time may be worth it [18].

The cost-effectiveness between the two methods has not been studied. Another drawback of the navigated system is that the cost may be higher than the conventional method. Novak et al. speculated the expenditure of the navigated system in TKA to be $\$ 1500$ for every patient [16]. We considered the following items ought to consider the aspect of expenditure, such as revisions, potential complications and functional outcomes.

The critical aspects for studies in the future:

(1) Further studies are needed to assess the long-term clinic outcomes of UKA. (2) The CT scanning might be more satisfactory because it offers a more precise assessment of the cement polyethylene. (3) Prospective studies are required to compare the hospital and socioeconomic cost-effectiveness between the two methods. (4) Further researches are necessary to determine the ideal target value of the tibial component. (5) More approaches, like the robotic systems, ought to 
be explored during the comparison between these 2 approaches.

Certain flaws ought to be taken into consideration. (1) The proofs of certain selected researches were not sufficient and merely two RCTs were selected herein, which might affect the accurateness of the gathered outcomes. (2) Great inhomogeneity of certain outcomes was discovered in the present gathering analysis. The diverse navigation systems, operative technologies, calibration of targets, component types, medial or lateral UKA, fixed or mobile bearing, cemented or non-cemented component and radiography measurement approaches were thought to be the causes. Nevertheless, our team failed to carry out sub-group assay of those elements owing to insufficient data. (3) Little research had adequate postoperation follow-up time, which was the primary cause hindering us from acquiring more stringent and persuasive results. (4) The specimen size of some selected researches was comparatively small, particularly the study of Konyves et al. (5) There are publication biases.

\section{Conclusion}

Based on our research, the navigated system provided better radiographic outcomes and no significant difference in the risk of complications with longer surgical time than the conventional techniques. But no significant differences were found in functional outcomes. Because the included studies were small samples and short-term follow-up, high-quality RCTs with large patients and sufficient follow-up are required to identify the long-term effect of the navigated system.

\begin{abstract}
Abbreviations
UKA: Unicompartmental knee arthroplasty; TKA: Total knee arthroplasty; OKS: Oxford Knee Score; KSS score: American Knee Society Knee Score; WOMAC score: The western Ontario and McMaster Universities Osteoarthritis Index; MA: Mechanical axis; ROM: Range Of Motion; HSS: The Hospital for Special Surgery; NOS: Newcastle-Ottawa Scale; RCTs: Randomized controlled trials; Cl: Confidence interval; OR: Odd ratio; MD: Mean difference.
\end{abstract}

\section{Acknowledgements}

Not applicable.

\section{Authors' contributions}

YQ and XK conceived of the design of the study. WQ and SJ participated in the literature search, study selection, data extraction and quality assessment. All authors participated in the design of the study and performed the statistical analysis. XK finished the manuscript. All authors read and approved the final manuscript.

\section{Availability of data and materials}

All data generated or analyzed during this study are included in this published article.

\section{Declarations}

Ethics approval and consent to participate Not applicable.
Consent for publication

Not applicable.

\section{Competing interests}

The authors declare that they have no competing interests.

\section{Author details}

'Department of Joint Surgery, Huangshan City People's Hospital, Huangshan, Anhui, China. ${ }^{2}$ Department of Rheumatology, The First Affiliated Hospital of Soochow University, Suzhou 215000, Jiangsu, China.

Received: 26 October 2021 Accepted: 9 February 2022

Published online: 24 February 2022

\section{References}

1. Mehranfar S, Abdi Rad I, Mostafav E, Akbarzadeh A. The use of stromal vascular fraction (SVF), platelet-rich plasma (PRP) and stem cells in the treatment of osteoarthritis: an overview of clinical trials. Artif Cells Nanomed Biotechnol. 2019;47(1):882-90.

2. Kang YH, Lee HJ, Lee CJ, Park JS. Natural products as sources of novel drug candidates for the pharmacological management of osteoarthritis: a narrative review. Biomol Ther. 2019:27(6):503-13.

3. Pourakbari R, Khodadadi M, Aghebati-Maleki A, Aghebati-Maleki L, Yousefi $\mathrm{M}$. The potential of exosomes in the therapy of the cartilage and bone complications; emphasis on osteoarthritis. Life Sci. 2019:236:116861.

4. Aletto C, Zara A, Notarfrancesco D, Maffulli N. Computer assisted total knee arthroplasty: 2.5 years follow-up of 200 cases. Surgeon. 2021;19(6):e394-401.

5. Suda Y, Takayama K, Ishida K, Hayashi S, Hashimoto S, Niikura T, Matsushita T, Kuroda R, Matsumoto T. Improved implant alignment accuracy with an accelerometer-based portable navigation system in medial unicompartmental knee arthroplasty. Knee Surg Sports Traumatol Arthrosc. 2019;28:2917-23.

6. Wang WJ, Sun MH, Palmer J, Liu F, Bottomley N, Jackson W, Qiu Y, Weng WJ, Price A. Patterns of compartment involvement in end-stage knee osteoarthritis in a Chinese Orthopedic Center: implications for implant choice. Orthop Surg. 2018;10(3):227-34.

7. Zhang Z, Zhu W, Zhu L, Du Y. Superior alignment but no difference in clinical outcome after minimally invasive computer-assisted unicompartmental knee arthroplasty (MICA-UKA). Knee Surg Sports Traumatol Arthrosc. 2016:24(11):3419-24.

8. Lonner JH, Klement MR. Robotic-assisted medial unicompartmental knee arthroplasty: options and outcomes. J Am Acad Orthop Surg. 2019;27(5):e207-14.

9. Grant AL, Doma KD, Hazratwala K. Determination of the accuracy of navigated kinematic unicompartmental knee arthroplasty: a 2-year follow-up. J Arthroplasty. 2017;32(5):1443-52.

10. Rosenberger RE, Fink C, Quirbach S, Attal R, Tecklenburg K, Hoser C. The immediate effect of navigation on implant accuracy in primary mini-invasive unicompartmental knee arthroplasty. Knee Surg Sports Traumatol Arthrosc. 2008;16(12):1133-40.

11. Saragaglia D, Marques Da Silva B, Dijoux P, Cognault J, Gaillot J, Pailhe R. Computerised navigation of unicondylar knee prostheses: from primary implantation to revision to total knee arthroplasty. Int Orthop. 2017:41(2):293-9.

12. Manzotti A, Cerveri P, Pullen C, Confalonieri N. Computer-assisted unicompartmental knee arthroplasty using dedicated software versus a conventional technique. Int Orthop. 2014;38(2):457-63.

13. Jung KA, Kim SJ, Lee SC, Hwang SH, Ahn NK. Accuracy of implantation during computer-assisted minimally invasive Oxford unicompartmental knee arthroplasty. A comparison with a conventional instrumented technique. Knee. 2010;17(6):387-91.

14. Saragaglia D, Cognault J, Refaie R, Rubens-Duval B, Mader R, Rouchy RC, Plaweski S, Pailhe R. Computer navigation for revision of unicompartmental knee replacements to total knee replacements: the results of a case-control study of forty six knees comparing computer navigated and conventional surgery. Int Orthop. 2015;39(9):1779-84. 
15. Jenny JY, Boeri C. Unicompartmental knee prosthesis implantation with a non-image-based navigation system: rationale, technique, case-control comparative study with a conventional instrumented implantation. Knee Surge Sports Traumatol Arthrosc. 2003;11(1):40-5.

16. Chona D, Bala A, Huddleston IIIII, Goodman SB, Maloney WJ, Amanatullah DF. Effect of computer navigation on complication rates following unicompartmental knee arthroplasty. J Arthroplasty. 2018;33(11):34373440.e3431.

17. Weber P, Utzschneider S, Sadoghi P, Pietschmann MF, Ficklscherer A, Jansson V, Muller PE. Navigation in minimally invasive unicompartmental knee arthroplasty has no advantage in comparison to a conventional minimally invasive implantation. Arch Orthop Trauma Surg. 2012:132(2):281-8.

18. Weber P, Crispin A, Schmidutz F, Utzschneider S, Pietschmann MF, Jansson V, Muller PE. Improved accuracy in computer-assisted unicondylar knee arthroplasty: a meta-analysis. Knee Surg Sports Traumatol Arthrosc. 2013;21(11):2453-61.

19. van der List JP, Chawla H, Joskowicz L, Pearle AD. Current state of computer navigation and robotics in unicompartmental and total knee arthroplasty: a systematic review with meta-analysis. Knee Surg Sports Traumatol Arthrosc. 2016;24(11):3482-95.

20. Konyves A, Willis-Owen CA, Spriggins AJ. The long-term benefit of computer-assisted surgical navigation in unicompartmental knee arthroplasty. J Orthop Surg Res. 2010;5:94.

21. Song EK, Mohite N, Lee SH, Na BR, Seon JK. Comparison of outcome and survival after unicompartmental knee arthroplasty between navigation and conventional techniques with an average 9-year follow-up. J Arthroplasty. 2016;31(2):395-400.

22. Downs $\mathrm{SH}$, Black N. The feasibility of creating a checklist for the assessment of the methodological quality both of randomised and nonrandomised studies of health care interventions. J Epidemiol Community Health. 1998;52(6):377-84

23. Stang A. Critical evaluation of the Newcastle-Ottawa scale for the assessment of the quality of nonrandomized studies in meta-analyses. Eur J Epidemiol. 2010;25(9):603-5.

24. Furlan AD, PennickV, Bombardier C, van Tulder M. 2009 updated method guidelines for systematic reviews in the Cochrane Back Review Group. Spine. 2009;34(18):1929-41.

25. Aldinger PR, Gill HS, Schlegel U, Schneider M, Clauss M, Goodfellow JW, Murray DW, Breusch SJ. Is computer navigation a usefull tool in unicompartmental knee arthroplasty? A pilot cadaver study. Orthopade. 2005:34(11):1094-102

26. Adulkasem N, Rojanasthien S, Siripocaratana N, Limmahakhun S. Posterior tibial slope modification in osteoarthritis knees with different ACL conditions: cadaveric study of fixed-bearing UKA. J Orthop Surg. 2019;27(2):230949901983628.

27. Chowdhry M, Khakha RS, Norris M, Kheiran A, Chauhan SK. Improved survival of computer-assisted unicompartmental knee arthroplasty: 252 cases with a minimum follow-up of 5 years. J Arthroplasty. 2017;32(4):1132-6.

28. Toda A, Ishida K, Matsumoto T, Sasaki H, Takayama K, Kuroda R, Kurosaka $M$, Shibanuma N. Inaccurate rotational component position in image-free navigated unicompartmental knee arthroplasty. Asia Pac J Sports Med Arthrosc Rehabil Technol. 2016;3:19-24.

29. Khamaisy S, Gladnick BP, Nam D, Reinhardt KR, Heyse TJ, Pearle AD. Lower limb alignment control: is it more challenging in lateral compared to medial unicondylar knee arthroplasty? Knee. 2015;22(4):347-50.

30. Seon JK, Song EK, Park SJ, Yoon TR, Lee KB, Jung ST. Comparison of minimally invasive unicompartmental knee arthroplasty with or without a navigation system. J Arthroplasty. 2009;24(3):351-7.

31. Jenny JY. Unicompartmental knee replacement: a comparison of four techniques combining less invasive approach and navigation. Orthopedics. 2008;31(10 Suppl 1):57.

32. Cossey AJ, Spriggins AJ. The use of computer-assisted surgical navigation to prevent malalignment in unicompartmental knee arthroplasty. J Arthroplasty. 2005:20(1):29-34.

33. Jenny JY, Muller PE, Weyer R, John M, Weber P, Ciobanu E, Schmitz A, Bacher T, Neumann W, Jansson V. Navigated minimally invasive unicompartmental knee arthroplasty. Orthopedics. 2006;29(10 Suppl):S117-121.
34. Keene G, Simpson D, Kalairajah Y. Limb alignment in computer-assisted minimally-invasive unicompartmental knee replacement. J Bone Joint Surg Br. 2006;88(1):44-8.

35. Lim MH, Tallay A, Bartlett J. Comparative study of the use of computer assisted navigation system for axial correction in medial unicompartmental knee arthroplasty. Knee Surg Sports Traumatol Arthrosc. 2009;17(4):341-6.

36. Perlick L, Bathis H, Tingart M, Perlick C, Luring C, Grifka J. Minimally invasive unicompartmental knee replacement with a nonimage-based navigation system. Int Orthop. 2004;28(4):193-7.

37. Valenzuela GA, Jacobson NA, Geist DJ, Valenzuela RG, Teitge RA. Implant and limb alignment outcomes for conventional and navigated unicompartmental knee arthroplasty. J Arthroplasty. 2013;28(3):463-8.

38. Saragaglia D, Picard F, Refaie R. Navigation of the tibial plateau alone appears to be sufficient in computer-assisted unicompartmental knee arthroplasty. Int Orthop. 2012;36(12):2479-83.

39. Jenny JY. Minimally invasive unicompartmental knee arthroplasty. Eur J Orthop Surg Traumatol. 2018;28(5):793-7.

40. Kennedy WR, White RP. Unicompartmental arthroplasty of the knee. Postoperative alignment and its influence on overall results. Clin Orthop Relat Res. 1987;221:278-85.

41. Pearle AD, van der List JP, Lee L, Coon TM, Borus TA, Roche MW. Survivorship and patient satisfaction of robotic-assisted medial unicompartmental knee arthroplasty at a minimum two-year follow-up. Knee. 2017:24(2):419-28.

\section{Publisher's Note}

Springer Nature remains neutral with regard to jurisdictional claims in published maps and institutional affiliations.

Ready to submit your research? Choose BMC and benefit from

- fast, convenient online submission

- thorough peer review by experienced researchers in your field

- rapid publication on acceptance

- support for research data, including large and complex data types

- gold Open Access which fosters wider collaboration and increased citations

- maximum visibility for your research: over $100 \mathrm{M}$ website views per year

At BMC, research is always in progress.

Learn more biomedcentral.com/submissions 